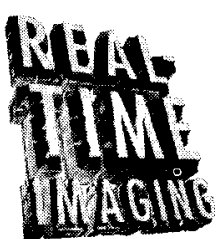

\title{
Negotiating the World of Make-believe: -The Aesthetic Compass
}

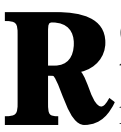

egardless of how far one stretches the definition of virtual reality (VR) (hopefully not so far as to negate its scientific and technological identity), the field is one of high expectations and of many yet unattained goals (technical, scientific, communicational). Some of its hardware (the headset, the glove) indeed became available at bearable prices (as promised by the early innovative entrepreneurs). Tools for building VR applications help in the design and implementation of new products deployed in hard-core science research or, at the other end of the spectrum, in wide-audience entertainment. Progress in the design and production of faster processors, better I/O (i.e., increased bandwidth and speed), and simple, more robust operating systems lead many to predict that the maturity of computer technology will be established in the high performance specific to the domain of virtual reality. However, an issue more significant to the ascertainment and viability of VR has yet to be approached: the crudeness, not to say ugliness, of its means of expression, in particular, of its visual component. The disparity between technological progress, culturally acknowledged formal qualities translated into VR components, and the ability to generate new expressive forms of distinction is undermining the field. The more one has the chance to experience new virtual realities, some extremely ingenious from a computational viewpoint, the more one fears that the medium is under the curse of submediocrity, at least in respect to its aesthetic condition. One of the theses of this article is that in the VR domain, as in any other form of human activity, the efficiency of the activity depends upon its underlying aesthetics. The degree of acceptance of new scientific and technological means, methods, and perspectives reflects expectations of human practice more than anything else. The aesthetic component, as difficult to formalize and encode as it is, affects the legitimacy of innovative endeavors, which VR definitely is, by ultimately affecting its efficiency. Hype and hope mix easily in reality, but even more in the realm of the virtual. The expectation of a strict definition of VR might seem an issue of academic interest only. But once we address the encompassing aspect of effectiveness in this field - effectiveness being its raison d'étre - we rapidly understand that specificity, i.e., precise scientific and epistemological identity, is reflected in design expectations and requirements for VR applications. These can be seen as instantiations of aesthetic exigencies. 01995 Academic Press Limited

Mihai Nadin

Department of Computational Design, University of Wuppertal, Germany nadin@wronx.uni-wuppertal.de nadin@math.uni-wuppertal.de 


\section{Introduction}

Virtual reality is (still) a new frontier in computation. Those not interested in the game of ever changing funding priorities of venture capital investment will argue that after all is said and done, VR is, if not more complex computer graphics, then at most a more elaborate form of interactive multimedia. Others, well aware that a new funding focus stimulates groundbreaking research, could easily show that with VR computing entered a new phase. As a non-command form of human-computer integration, VR is an example of many transcended limitations: no more pervasive WIMP user interfaces (i.e., no windows, no icons, no mice, no pointing), at least not within the desktop metaphor; no more user-interface tools (graphical or otherwise), rather an integration of the interface in the application; no more unity and homogeneity of data types; no more files. The list is far from complete. Moreover, the list is not clear-cut and cannot be exactly because we know VR more by examples than by (or through) a coherent and systematic theory. And examples are as opportunistic as funding priorities or marketing and investment considerations. Even the socalled serious applications -design and architectural walk-throughs, maintenance simulation of complex machinery (aircraft, satellites, submarines), therapy (in various areas of medical care), training, molecular modelling and docking of pharmaceuticals or new materials, learning, and so on - do not necessarily lead to one comprehensive definition. (Figures 1 and 2).

Very early on, VR was identified with its specific I/O paraphernalia: helmet (with stereoscopic eye goggles and headphones) and the data-glove; then with the immersion of the individual (no longer really a user) in an interactive new 'reality'. Now it describes a new interface [CAVE, e.g. (1)], augmented reality - actually the super-imposition of digitally processed information over the real world we look at-remote control of robots or processes (tele-presence), and 3D imagery and sound presented in open-ended forms eliciting viewers' reactions (change the script as the plot unfolds). Whether in the form of a virtual brewery (with a virtual tour guide), a voyage through the countryside or on a sailing ship (as during prohibition, with the aim of smuggling whiskey), a shopping trip or a game, such and other applications can be quite complex and computationally very demanding. Even rather simplistic CD ROM-based games (for children and for adults), cybersex experiences (with goggles), not to mention telecommuting (work on the

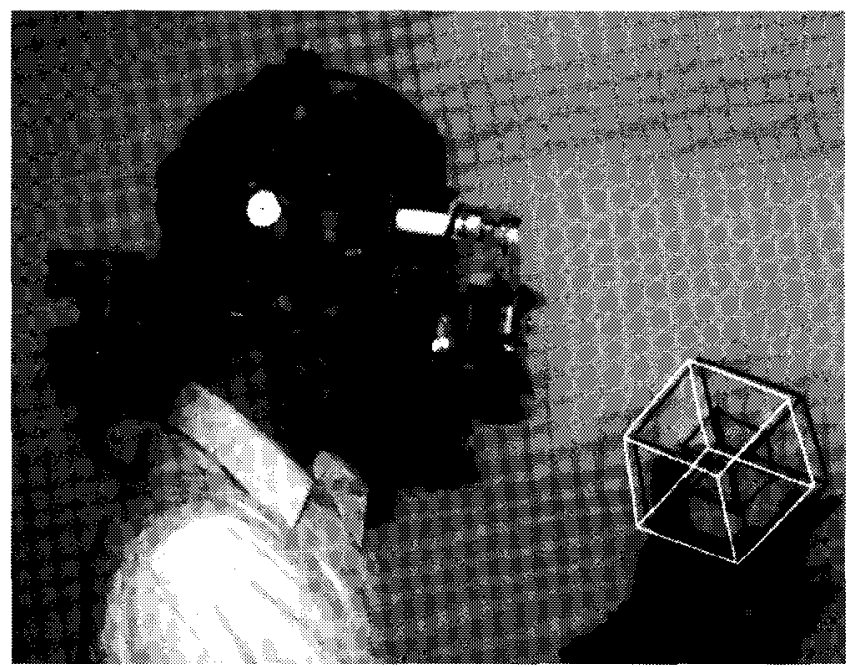

Figure 1. VR gear unmistakeably identifies the subject. In this image from the NASA Advanced Displays and Spatial Perception Group, an attempt was made to identify what the subject sees. Manipulation of a 4D figure, i.e., a hypercube, (sections are cubes) requires focus on geometric representations. The underlying aesthetics of such representations is based on proportion, vanishing point perspective, contrast.

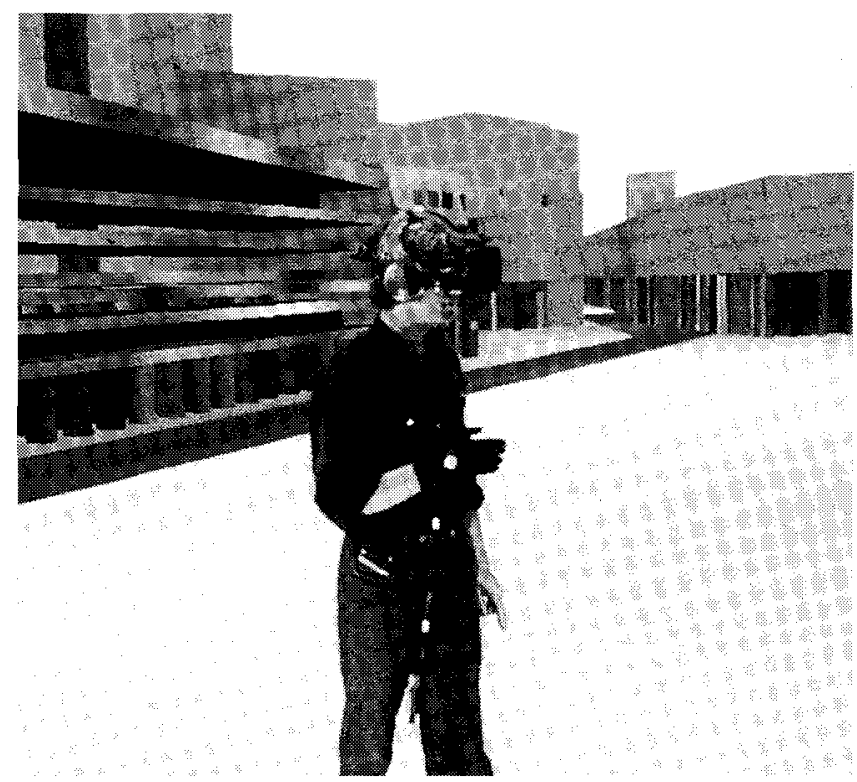

Figure 2. Cybercity-virtual reality application from Art + Corn, Berlin, Germany. Immersion in a virtual city landscape- this is the subject of the image. The underlying aesthetics is that of a dynamic, i.e., changing perspective. The major computational effort is that of providing a sense of scale (of the new environment), not an impression of detail. 
information grid, and the emergence of the virtual office) are qualified as forms of VR, and not only in media reports.

\section{Art and Science}

Paradoxically, not only did the thought of VR originate, partially at least, in the realm of art Myron W. Krueger's 'artificial reality', as well as William Gibson's 'cyberspace' testify to this- but it most certainly stimulated many attempts in areas of artistic focus. Where computers made it more convincingly in art's bureaucracy and commerce than in the creative domain, VR, although still in its infancy, was already showcased at the Guggenheim Museum (2) and promises. to receive ever larger public exposure. The movie industry, for instance, is very actively pursuing projects that will advance the art of filmmaking as well as the technology. Even today's most spectacular 3-D Imax projections-in a way a product that integrates VR elements (the wrap-around goggles fitted with infra-red sensors, liquid crystal lenses, and stereo headphones, for example) - would look primitive when the interactive movies of virtual worlds eventually open to the public. Networked VR will change not only the traditional access to art, but also the act of creation as well. There is art in science, embodied in the elegance of expressions, harmony of means (such as the symbolism of scientific notation), rhythm of successive arguments, contrasts between valid and false inferences. And there is science in art, embodied in the rationality of the form, the logic of colors and patterns, the clarity of meaning as ascertained by the artist or conjured by the audience. The realm of VR experiences corresponds to a new type of cognitive activity. Its object is the constitution of a new space and time for interaction. As we enter this fascinating realm, the art of science and the science of art are redefined.

Jaron Lanier - the erstwhile evangelist for virtual reality (the person who contrived this oxymoron) and an exceptional musician - addressed the Reality Club (February 28, 1990) in New York, selecting real time as definitory of the new world of images and sounds in which the human is immersed via computerized clothing. In his definition, real-time image and sound, and whatever else makes up the virtual world, i.e., the art of interaction, stand in opposition to the canned or prerecorded. This new world is made anew each $1 / 20$ of a second- at least in Lanier's projects. Turning one's head in the real world results in the real-time 're- writing' or 're-making' of the virtual world in order to compensate for the new position of the eyes and ears. The hand affects even more in this environment as it 'grabs' objects and moves them, or as it tries to perform other functions, such as playing a virtual instrument (Thomas Zimmerman's 'air guitar' of VR's early days, for example). Krueger imagined VR as a 'responsive environment' (3) in which 'the aesthetics of interaction will be as important as the efficiency'. Stephen R. Ellis described it, some 10 years later, as 'head-referenced computer displays that give the users the illusion of displacement to another location' (4). Illusion, without which VR does not exist, clearly belongs to the realm of art. In order to generate illusions (of presence, movement, qualities, etc.), VR practitioners looked at the many techniques that artists developed over time and started to mimic some of them. Along this line, programmers in our days see new challenges in finding algorithms for shifting the virtual world as the subject turns his head. Or, better yet, they took up the challenge of writing code that maintains the coherence between the virtual environment and the physical world.

This goal of maintaining coherence is more abstract than that of creating the illusion of perspective. But both are aesthetic in nature. Artistic representations (realistic, surrealistic, abstract, etc.) are subject to meaningful interpretation only to the degree that they are coherent with the context in which they are interpreted. Space representations in flight simulators are as successful as the level of coherence achieved in the virtual experience. The question, however, is: can aesthetic requirements be effectively translated into scientific and technological goals? Moreover, should the problem-solving process of technological innovation be guided by aesthetic awareness resulting from a methodic foundation, let's say in art or design? Or will this aesthetic awareness automatically follow as technology will afford it? Or, even, will a new aesthetics emerge?

As things stand, system designers break the problem into what is possible (for instance, how much realism can be generated with our graphic engines), and what remains takes the form of so-called 'bells and whistles'. The possible is 'translated' into expectations of necessary chip cycles, speed, band-width, multitasking, and other computing characteristics. More often than not, they use common sense or infer from their individual likes and dislikes. Interface engineers handle I/O problems caused by ever increasing varieties of data 
and the more critical aspects of synchronization peculiar to coordination of image, sound, haptic and other sensory components of the virtual world in a rather intuitive way. Many more, at work in handling complex tasks of modelling, rendering, and real-time animation, rely on mediocre 'how-to' (paint, compose, animate, etc.) literature or continuing education materials. The simulation component, far from being the sum total of such tasks, emerges as even more heavily handicapped by lack of aesthetic discipline.

Addressing a different cognitive level, i.e., that characteristic of the more complex experience of motion, simulation requires the advanced knowledge and aesthetics of dynamic representation. What connects the input provided by sensors (for head position, movement, etc.) to the output, i.e., stimulation of senses by effectors (for display, sound, tactility), are complex programs (for simulation, telecommands, data processing) running on and to special purpose hardware. The design of such programs is extremely demanding not only in terms of computational requirements and algorithmic thinking, but also from the perspective of the expressive means through which change is represented. Simulation, for which we are still ill prepared, even after quite an extensive experience with flight simulators (preceding and anticipating VR), is probably at the core of this new computing frontier. The credibility of any simulation reflects foremostly its aesthetic appropriateness (Figure 3).

And if all this does not add up to enough complexity, imagine the real (sic!) virtual reality: not one, but many individuals interacting in this synthesized world. Moreover, not only graphics and sound, but information addressing all the senses, that is, a world in which the analog and the digital meet. And what results is an unfolding of new realities in real time. Technically, this requires the integration of digital and signal processing (DSP chips, in particular), i.e., of heterogenous data. With all this in mind, I was not surprised to learn that even the most awarded computer graphics educator,
Andries van Dam, by nature an optimist when it comes to computation, is sceptical in regard to how present hardware can meet the demands of VR (cf. Symposium Research Frontiers in Virtual Reality, San Jose, October 1993). Furthermore, he and others noticed that software for generating real-time artificial environments lacks speed, is difficult to implement, rigid, and is not really extendible. As more and more research laboratories and commercial facilities promise better development tools and production environments, we still torment ourselves with what the first entrepreneurs made available in the form of so-called toolkits (among them, PROvision, World Toolkit, Reality Built for 2, Microcosm- all trademarks). The goal of getting a better handle on concurrence, never mind developing generic models and, especially, bridging between what a VR world suggests as possibilities and the openendedness of human interactions within such worlds seems at times even more elusive than it was 3 to 4 years ago.

\section{The Logic of Senses}

If, for all practical purposes, this is the current state of affairs in VR, isn't it premature or indeed foolish to even attempt to address its aethetics? The major thesis to be detailed in this article is exactly that investigating, designing, or producing multi-sensory environments (virtual or those characteristic of multimedia) requires as much scientific and technological knowledge as it requires awareness of the elements constituting the underlying aesthetics of such environments. As the logic of the senses [in the definition given by Baumgarten, who coined the term in his work Aesthetica, 1750 (5)], aesthetics is constitutive, not illustrative, of human interaction, including our relation to reality. Some scientists and technologists might discard this statement as merely philosophical. Still, as long as they built their own domain on a foundation of logic (Boolean or otherwise), it would be unwise to ignore the

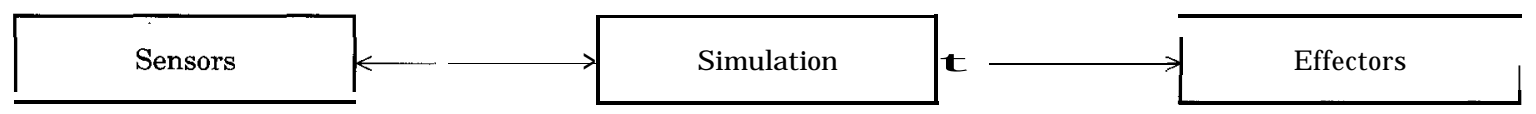

Figure 3. The core of virtual reality. Reactions, alternative decisions, and even planned actions of a VR user result from the interplay of sensors and effectors and are reflected in the state of the simulation. High performance image generation in real time, as well as sound processing (3D or convolved) and interfacing with various input and output devices are tightly integrated. 
consequences of inappropriate aesthetic decisions for optimizing the computational endeavor.

The experience of computer graphics enlightened many computer scientists in regard to aesthetic considerations and criteria for evaluation. Animation helped further; 3D modelling and rendering were literally impossible without acknowledging the cognitive mechanisms based on aesthetic processing that allows designers to imagine new objects or processes and effectively design them. On the desktop publishing screen, many learned the aesthetics of typography and understood that producing a book or a newspaper is as much a cultural accomplishment as it is a technological feat (think of the new plateless printing). Production can be quick and economical, but if the product is ugly, it is useless. Granted, the word 'ugly' stands for physical appearance, appropriateness of type and layout, print quality, harmony (or lack thereof) between paper, ink, binding, and some other well defined aspects of a book. Similar to the situation in science (mathematics, physics, biology, etc.), much of what was accomplished in the aesthetics of computation until now reflects sensitivity and understanding of aesthetic value that form the context of our culture. And as with ah there is -from infant lifeforms to technology and scientific theories - beginnings imitate what is already established. Computer graphics, animation, and computer music emerged as emulations. To a great extent, they still imitate artifacts and art works that seem to have established a model, an ideal. The emulation of aesthetic qualities results also from the dynamics of active learning, i.e., of learning by example.

With the maturity of computation, aesthetic awareness can be pursued beyond emulation. In order to do so, we need to account for the variety under which aesthetic experiences unfold in the context of technological advancement. Graphics elicited the underlying aesthetics of two-dimensional images. Symmetry, order, rhythm, counter-space, and much more in that vein, encoded in the culture of images preceding computer graphics we were exposed to, came into focus in a context of digital processing. In an effort to deal with jaggies, flatness of shapes, monotony of repertory (the world seemed to be made up of always shiny circles, squares, horizontals, and verticals), we learned how to integrate aesthetic expectations characteristic of other media. From images on paper or canvas, as media for artistic expression, we learned how to derive a new aesthetics. This new aesthetics corresponds to a medium of technological constraints that seemed unbearable to anyone who ever saw a drawing by Dürer or admired the elaborate art of Japanese silk-screen printing.

What I am trying to express here is that the underlying aesthetics leads to different embodiments, to characteristics of different types of visual expression. These embodiments correspond to the variety of human experiences - from farming and unskilled or skilled labor to scientific and humanistic research, to art and design. The aesthetic characteristics of the early plough were very closely connected to its functional attributes. But as progress was made by replacing wood and stone with metal, and oxen with engines, the initial functional structure has been preserved while the aesthetics was continuously improved. Take, for that matter, any other example-from the first car, airplane, microscope, etc. to their current embodiments. The intention is not only to make the plough more beautiful or the car or airplane more attractive. The process is one of optimization in view of the many requirements of human activity. Each time such a process takes place, it confirms that aesthetic characteristics affect functionality and performance. The process also corresponds to acknowledging the variety of contexts in which such experiences take place. The precious book of the past reflected in its aesthetics a relationship of permanence between the reader and the text. Under current circumstances of rapid turnover in knowledge, shorter production cycles and much shorter interactions, the permanence expressed in the illuminated manuscripts of the past is unimaginable. Medium - in this case, the book - is only one example of how the underlying aesthetics, reflected in some very well defined qualities, is connected to the characteristics of practical activity. Images on a CRT screen or LED display are meant to accelerate, not to hinder, the rhythm of human activity. The 'electronic book' of our age does exactly this, obviously no longer emulating the books of the past (Figure 4).

The underlying aesthetics is the unifying element of such accomplishments as the architecture, painting, sculpture, music, poetry, and dance of the Renaissance or of the American hyperealism (to name only two examples, quite remote in time and unrelated in their characteristics). In the succession of styles, the underlying aesthetics ensures, as does Boolean logic in other ways, that people, can understand each 'program', identify goals and means, and judge how successful the effort is. Adequacy of aesthetic means results from an effort to optimize the process or the product not dissimilar to optimization of code. I submit this analogy 


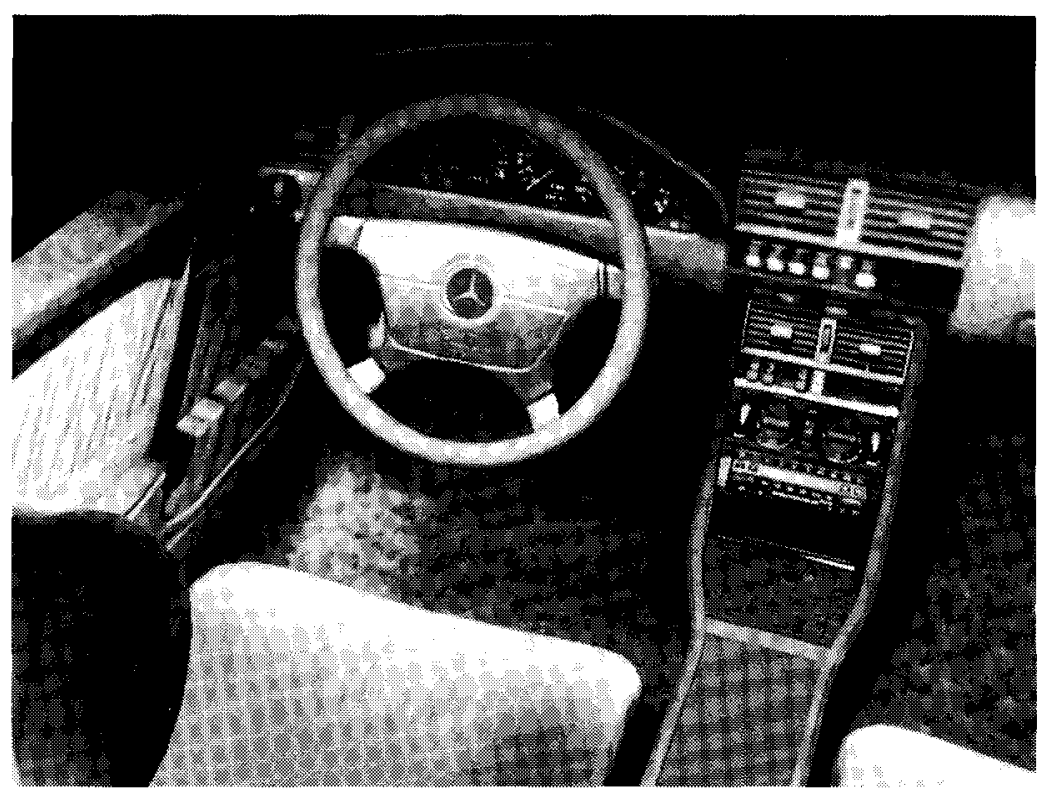

Figure 4. Daimler 2 (Art + Corn, Berlin). In modelling new products, VR techniques facilitate design alternatives. Aesthetically, the effort is directed towards detail. Accordingly, the computational effort is in the area of expressive, high precision rendering.

between an aesthetic program and software programming on purpose in order to make sure that the barrier of specialization (WE know computers, THEY know art; we give THEM programs, they give US aesthetic pleasure) does not prevent the possibility of understanding the subject under discussion. The static image is subjected to a logic of our senses, i.e., aesthetics-of seeing, touching, hearing - quite different from that of a dance, a musical event, a sculpture, or a landscape design. The underlying aesthetics of an equation in mathematics or physics, of a chemical or biogenetic formula, might reflect symmetry and order, but also a sense of economy of means. Aesthetics will not be the final proof of any theorem. But as we learned from many scientists, it will confer upon their formulae an expectation of legitimacy. The same applies to visualization, which transcended computer graphics and the obsession of realism and made computational science finally possible. Epistemologically, this is yet another way of ascertaining the role that aesthetic considerations play in our attempt to optimize the expression of truth and knowledge. Occam's razor-no need to multiply entities beyond necessity - applies very well in this realm. That real-time computation requires a qualitatively different set of aesthetic identifiers cannot go unnoticed, especially in view of the fact that VR is more than real-time imaging.

\section{Types of Representation}

The requirement for defining what is characteristic of VR before even attempting to address its aesthetic condition reflects the acknowledgment of the variety through which the underlying aesthetics influences the effectiveness of human experiences. While VR cannot directly benefit from the awareness of the aesthetics of typography, for example, or from awareness of the aesthetic condition of scientific theories, it seems that it can benefit from the experience we accumulated in human activities involving syncretism, or involving all our senses. Indeed, the attempt to generate in real time a three-dimensional world of images, sounds, textures, and other sensory substitutes for the richness of the physical world conjures up more than what we know from art, equations, print communication, or even television. It refers to what is alive and unfolds as living. As we know from history (and its post-modern representations), the closest people came to emulating the underlying aesthetics of natural events was in various forms of rituals, including rituals of a scientific or technological nature. In more than one way, virtual reality experiences have a ritualistic dimension. Within a VR environment, the subject relates not to nature, but to dynamic representations reminiscent of past objects or actions pertinent to them. While nature, to which we 
still belong even in the VR experience, is void of symbols - signs of human convention -rituals are driven by a symbolic force.

In order to understand what this means, a short explanation needs to be provided. As zoon semiotikon (i.e., semiotic animal, as Felix Hausdorf, the reputed mathematician of the 19th century defined the human being), we handle a limited variety of representations. The most direct are iconic, representations based on likeness, as in the desktop metaphor and its familiar icons. Others are indexical, marks left by the object 'represented: animal footprints, the position of the windsock, indexical of wind direction, the data representing the head position in a VR application. And others -cognitively the most challenging and rewarding - are symbolic, that is, conventions such as those in language, in scientific formulae, in artistic expression (6). Within iconic representations, which have been predominant in computer graphics and are now in the emerging field of VR, the final obsession is realism and its associated aesthetics. Obviously, this aesthetics is not necessarily the same as the aesthetics of symbolic representations. Under certain circumstances, cognitive processes characteristic of the symbolic level of human activity can be negatively affected by submitting symbolic representations to an aesthetics of realism. Incidentally, in his unfinished book on the foundation of aesthetics, Baumgarten dedicated a chapter to semiotics. But it was not until Charles S. Peirce, the paragon of American science and philosophy, that a logical foundation was given to the theory and application of signs.

All these considerations (even the historic pointers) are submitted here as an argument for how difficult the problem of VR aesthetics is. If one feels discouraged looking at the sometime disappointingly poor images extracted from VR experiences, or facing them in a VR experience, the explanation is that even in VR good intentions pave roads to aesthetic hell. In addition, as already stated, the aesthetics cannot be improved after the VR context is created, i.e., after decisions regarding the nature of representations used have been made. They have to grow together, intertwined, in order to facilitate the much desired effectiveness of the experience.

Michael Heim (7) juxtaposed the Webster dictionary definitions for 'virtual' and 'real' and arrived at the following result: 'Virtual reality is an event or entity that is real in effect but not in fact'. If we add that what makes it possible is real-time computation and appropriate strategies for representation, we have a pretty good framework for further elaboration on the difference between effect and fact. Rich in information of all kinds, facts and effects are differentiated in human practical experiences in more than one way. Aesthetically, they define two poles: objectified aesthetics -patterns of shapes, colors, rhythms, contrast and harmony, symmetry, etc., appropriated from nature in the process of self-definition of the species; and subjective aesthetics - new patterns of formal qualities projected on life, work, designs, and products. Over time, the aesthetic patterns constituted perceptive cues and were accumulated in the cognitive endowment of the species. This is how, at a biological level first, and progressively at a social level, the aesthetic elements constituted an existential matrix. When I refer to underlying aesthetics, I have in mind this formative network, this powerful 'mold' and 'compass' at the same time. It is formative insofar as it results in individual patterns of perception. It is a mold since what we do carries the imprint of aesthetic awareness. And it is a compass in the sense of setting a direction, recognizable through choices we make among many possibilities of different aesthetic quality.

When moving from the known to the unknown, from the usual to the unusual, the individual, as well as the species, relies on the integrative power of the underlying aesthetics of iconic representations. We experience the world through the filter (eyes, ears, nose, touch sensors) of our culture, in which the underlying aesthetics crystallizes or, better yet, is recognizable. Our effectiveness in the world is affected by it. Throughout history, the better tools, the better forms of shelter, the better instruments for observation, as well as the more powerful scientific theories always proved to be those that were more aesthetically appropriate. Selection, whether natural or made by humans through axiological processes, confirmed aesthetic appropriateness as a criterion for viability and survival. Consequently, we have not only artifacts of all kinds, from weapons to bridges and cars and homes and scientific theories, to prove it, but also the acquired awareness of the role played by aesthetic factors in the dynamics of change. In some ways, authors of genetic algorithms that model natural selection are intent on integrating the aesthetic dimension in grading among design choices. When implemented in VR products, such genetic algorithms provide new means for achieving and maintaining aesthetic integrity. 


\section{Types of Interpretation}

The basic types of representations (iconic, indexical, symbolic) having been defined, the various ways representations are interpreted also need to be acknowledged. In some instances, representations are considered independent of the object represented, i.e., as entities characterized by the relations among their constitutive elements. For example, words, images, or sounds can be analysed syntactically and inferences made regarding successive letters, colors, or tones. Similar to iconic representation, the syntactic level is at the bottom of cognitive activity. To a certain degree, the infancy of computers ('brute force') and the respective computer science correspond to the effort to focus on syntactic aspects. Once the question regarding the relation between what is represented and the representation is raised, we enter the domain of the semantic. It is cognitively a more complex level since semantic considerations are more ambiguous than the syntax. (After all, the syntax can be right or wrong, i.e., true or false.) A VR application based on the aesthetic conventions of realism embodies in its 'description' of the world synthesized in real time, the expectation of semantic interpretation: 'Ah, this is the hand!' one discovers while moving his or her own hand while wearing a glove. 'And these are stairs, doors,. ..' This recognition takes place at the semantic level and is 'reflected in the computational effort to provide sufficient detail information.

But the most demanding dimension-the top level, cognitively- is the pragmatic: What are all these representations for? Computer graphics images of molecules are as good as classical renditions or illustrations, as we know them from books. But in the VR application, especially where haptic feedback (through ARM, i.e., Argonne Remote Manipulator, originally used, as we know, to handle radioactive materials) is integrated, the expectation is not a nice image but action upon the molecules, that is, pragmatics. Henceforth, aesthetic means, for example, those characteristic of animation, have to ensure that what is possible-the investigation of the docking of molecules -is made known to the user, the pragmatic interpreter. The computational effort is thus no longer one of modelling and rendering (with questions regarding the aesthetics of pseudo-coloring), but of dynamic simulation of intermolecular forces.

With all this in mind, we need to take a closer look at the various forms through which VR is made available.
An expectation of effectiveness is correlated to each type of application, i.e., to each type of representation used in particular cases. Expectations of effectiveness are not unequivocally defined. Is effectiveness the impression of reality that one experiences on a virtual bicycle? Or the ability to maintain one's balance? Or the number of CPU cycles used to create the illusion? Realism, immersion, computational performance, to mention only the most evident elements, belong to the equation of effectiveness. Accordingly, until we define what the optimal representation is in regard to the goal pursued, it is rather difficult to see how the aesthetic factor or the computational performance can ultimately affect it.

$\mathrm{VR}$, as a medium for communication, in particular as a visualization technology, is effective in ways different from those characteristic of VR as a medium for training, or for VR-assisted medical surgery. The VR experience of a walk through a future building or a journey on highways requires iconic representations, i.e., realism. The experience of visualization of molecules actually requires a symbolic representation and a different underlying aesthetics. In games or in flight simulators, indexical elements (describing position and speed) are dominant. The practical consequence is evident: the computational effort within available means of real-time imaging has to be focused on what is essential for the type of application. High resolution images are not necessary in the world of symbolic representations. In turn, games require high performance in tracking and associated aural channels. The potential medium of human interaction that VR techniques made available is different from the medium of scientific inquiry it makes available in applications of a cognitive nature.

Regardless of these distinctions, VR remains well defined by its generic condition of real-time generation of effective means for simulating the behavior of complex systems (Figure 5).

Despite the variety of applications, the majority of known VR programs is still designed on a semantic premise, not on pragmatic considerations. Likeness remains the goal and is an aesthetic identifier: use fractals for showing mountains, genetic algorithms for flowers and sea shells, various signal processing techniques for generating sound simili of birds 'singing', or of jets breaking the sound barrier. As a testimony to the power of aesthetics, since nature is by and large a rather widely shared reference, the aesthetics of imitation 

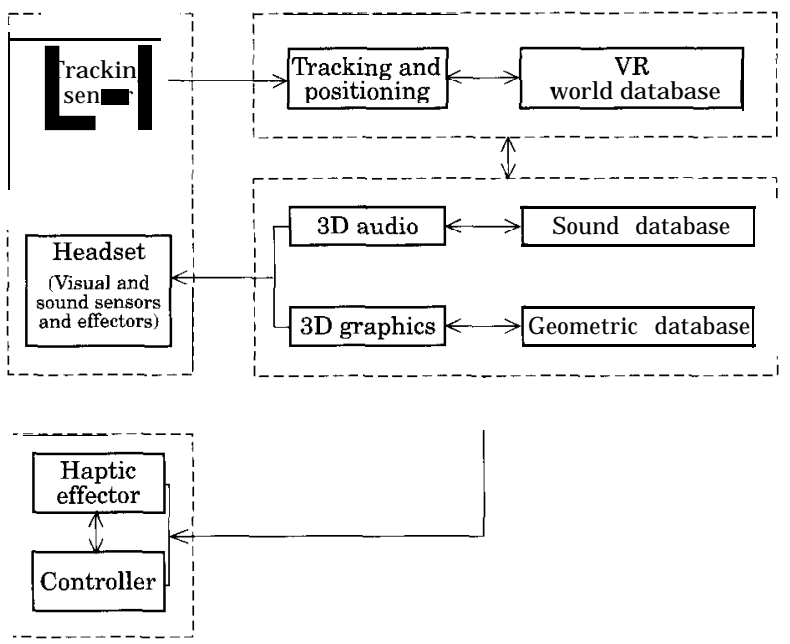

Figure 5. From a collection of components to a dynamic configuration. Four basic processes make up a real-time virtual system: the VR application (functional aspect), culling, transformations, and rendering. An application is a sequential succession of processes, the output of one being the input of the following process. The VR system cycles through once per frame for each image of the virtual world to be generated.

(mimesis) has already resulted in pretty impressive feats in each of the identified components. The epistemological problem is that the reference in VR is no longer nature, but our knowledge of various phenomena, knowledge expressed in computational form. What VR offers is not the photograph or the film of reality, but a dynamic configuration that will become alive only in action.

Indeed, VR transcends the semantically driven computational endeavor in order to reach the pragmatic level. Syntactic elements or semantic features are present, but integrated in a whole, a dynamic Gestalt. They are subordinated to the pragmatics of the elements making up the virtual world. Essential is not the appearance, but the functioning of elements, the synchronization, the simulation. Features of VR can be analogous to real-world experiences, but they can be 'non-natural' as well. Granted, in order to generate in real time the world that makes the VR experience possible, many previously developed resources can be used, and indeed are used. Let's face it, motion simulators go as far back as the late 1930s teleoperations have existed since 1940. Still, features originating from them are used today. So are geometrybased features (points, lines, regions), as well as texture-based features (defining surface roughness or smoothness, or edge density), first developed in com- puter graphics. At the beginning, hundreds of polygons or a thousand per second in describing objects in space were all that VR developers had. Until recently, a few hundreds of thousands of polygons per second made for an acceptable performance. Then the threshold moved to 500,000 (and the machine delivering it was called a Reality Engine), or 1 X 10".

Obviously, in many applications the expectation is realism, an aesthetic qualifier that does not reflect the technology used, but rather how successfully this technology, working on entities in the iconic domain, makes us believe that what we see is real. Massive parallelism now affords 5 X lo 6 Gouraud-shaped triangles per second (this is an intensity interpolation shading, developed in 1971 by Gouraud) and more than $4 \mathrm{X}$ lo 6 Phong-shaded triangles per second (this is a normal vector interpolation shading developed by BuiTuong Phong in 1975) with specular lighting and phototexturing. The refresh rate improved, too: 30 frames per second, supporting relatively smooth motion, can be expected on most platforms. Smoothness of motion (i.e., low lag values), not unlike that of cinematographic or video images, is a necessary aesthetic condition for the VR make-believe goal. And still, despite usage of such advanced technology, field use of viewing devices, not only those cheap helmet displays that inundated the market, can be difficult, disorienting, and moreover nauseogenic. Which says that we have to address, in addition to the necessary aesthetic condition of realism, the so-called sufficient aesthetic means, i.e., those directly affecting the biological condition of the human being immersed in a world where his or her own naturality is challenged. After all, if we pay attention to the unity between means of representation, desired interpretation, and the associated underlying aesthetics, we might discover that the answer to the many problems faced in VR development is not necessarily a matter of a $1000 \mathrm{MHz}$ chip or of slow transmission of tracking data, but of a better understanding of the cognitive aspects of human activity. Among these aspects, aesthetic characteristics play a major role.

\section{A W orld of Ideas}

I know of no better representation for the subject of VR than the drawing by Oskar Schlemmer entitled 'Der Mensch im Ideenkreis' (The Human Being in the Circle of Ideas, 1928) (Figure 6). 
Those concerned with addressing the many dimensions of human existence pertinent to VR might find more than aesthetic reward in carefully considering this drawing. In what follows, I shall 'paraphrase' Schlemmer's image.

VR data, in the end expressed in arrays of numbers, has as its source various sensors: for position, pressure, (sensation of grasping things that correspond to real objects, even though they are illusory), speed, direction. Information, as the drawing suggests, travels via the nervous system to the brain. The entire body (anatomy, physiology, psychology) is involved in the process of abstracting ideas. The scale of the being relates to the scale of the environment. The notion of affordance, introduced by J. J. Gibson (8) almost 60 years after Schlemmer's image was first seen, is translated in physical qualities corresponding to the relationship between the individual and the environment. Please

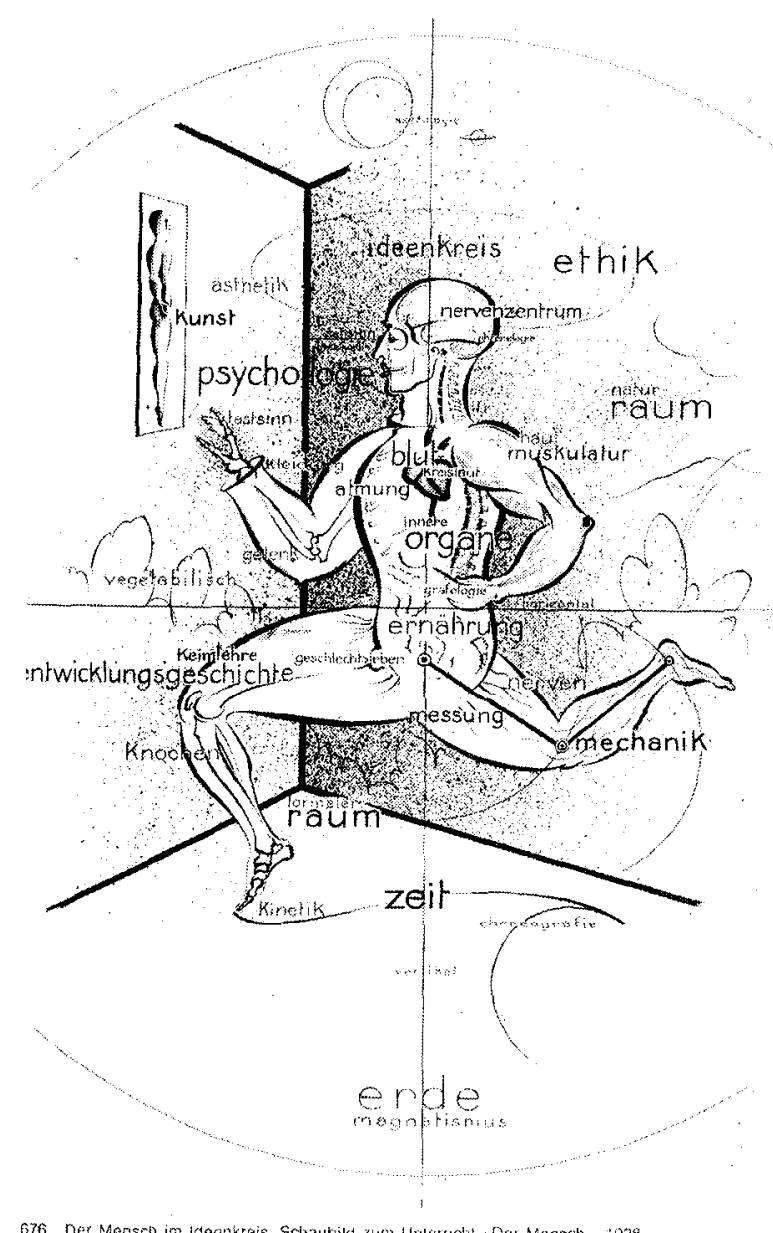

Figure 6. Oskar Schlemmer, 'Der Mensch im Ideenkreis'. take note that body mechanisms, sexual life, nutrition, ethics, and history (even what today we call superstitions) are part of this model.

As a noncommand type of computation, VR generates families of possibilities around an abstract model of the human being. What is returned in VR is not one of the values stored in the cubicles of a database, but a virtual object and its 'rules' for functioning. The abstract human being of the model is instantiated by the real user. Accordingly, what in the semantic realm prior to VR was the fundamental focus on encoding aesthetic rules in programs and decoding the meaning of graphics, animation, synthesized sound sequences or combinations thereof, becomes in VR the need to aesthetically and functionally make-believe. In fact, VR is a world of ideas, digitally driven, animated by the individual or individuals entering it.

The problem of effective make-believe is not trivial because as things stand, VR is not only an oxymoron as an expression, but also as a construct: how to override the user's 'natural' senses (which was Ivan Sutherland's goal; cf. 1965 lecture at IFIP on the Ultimate Display) while interfacing them to VR through sensory I/O devices. In a way, theater, and moreso the experiences of Cinematography-Cinerama and omnimax are reference display solutions - and television, attempted the same. Stereoscopic glasses were designed in order to provide the stereo image resulting from the superimposition of two flat images. No surprise, then, that some of the most successful ideas regarding how to make good VR applications come from either people who know something about theater research work on theater was taking place at the same time as research into simulation (9) or from people who revisited film theories (suspension of disbelief comes from the group that created CAVE) (1). So, can we look for answers to the very complex questions of VR in the aesthetic theories or practice of theater? Of film? Of television? Sure, but only if we understand that the situation is literally upside down: the actors in VR who make us believe are not other people (the actors) but ourselves. Moreover, the context in which make-believe has to occur and be continuously consolidated requires the aesthetics of identification in a medium that, in order to come into existence, requires alienating means: the head track display, the sound channels, the glove. The distinction between what is real-all these cyberspace elements- and what is 'play' - the experience in VR- raises many questions in the minds of those seeking the immersion. 
As attractive as ideas coming from theater, film, and video can be in helping those who design VR applications to understand the relationship between computation and underlying aesthetics, they actually are no more than sensitizers. More precisely, their domain-theater, film, and by extension television -is orthogonal to creating artificial immersive environments. Not others are the actors- as in Stanislawsky's or Meyerhold's theories of theater. And the purpose is not to have actors make us believe whatever they act out in a fixed frame of reference (the play takes place in a given setting). The VR application is an unwritten drama (or comedy) unfolding computationally on a dynamic stage. The research scientist, the surgeon, the individual trying to learn new skills, the remotely placed interacting persons are the 'writers' and 'actors' of the play, and the editors of what they see, hear, and feel. Some fragment of reality, let's say molecules of synthesized materials, when seen under the electronic microscope in the physical world is still part of reality. A real-time generated simili of a new molecular design is as much a simili as the stage design depicting a royal palace, a lunar landscape, or someone's bathroom. One can be stored on a hard disk, the other in a storage facility. The difference is in who will endow this dead world with life and how. Actors and playwrights do it for theater; actors with very different skills and scriptwriters do it for film and television. In VR, we expect this to happen as the immersed individual 'writes' a script and 'interprets' it at the same time. This is a medium of almost endless choice and unpredictable results. In the play, Romeo and Juliet eventually die. VR is open-ended, even though it is based on a limited number of constitutive elements ('alphabet'). As a matter of fact, the rules for generating the virtual world remind us of generative grammars, and I believe that these will be eventually applied in this field as means for handling dynamics. With all this in mind, we have here at least three different issues to address:

(i) how to establish a convention for representation;

(ii) how to support open-ended interactions;

(iii) how to allow for the suspension of the game.

\section{The VR Convention}

The first aspect is cognitively anchored in the wider realm of extending the experience of living and working in a given physical world to new experiences in an artificial reality. Some of these new experiences might confirm physical world constraints (e.g., as related to progression of time, gravity, perspective, 3D spaces, etc.). Others might as well challenge them (e.g., an artificial reality in which the future comes before the past, as in playback; things 'fall' up; perspective with more than one focal point, spaces of more than three dimensions, etc.). Regardless, the focus is on how the convention representation is established. As we have already seen, likeness (or iconicity) dominates at this level. Indexicality supports the effort. But the purpose is the new convention, which is in the end embodied in the VR symbolism. A bungy jump is an experience in the physical world; it takes place between t1 and t2 (time interval), from an altitude that can be defined, and it involves several artifacts meant to preserve the life of the jumper as well as to magnify the sensation of risk in free-fall. The underlying aesthetics is that of the natural environment. Few, if any, would go for a bungy jump over an ugly dump-site or under circumstances (rain, fog) affecting perception of space and time, of colors, perspective, depth. The event itself maximizes living at the borderline between pedestrian existence and daring challenge of physical laws against the dramatic background of natural beauty. A VR bungy jump, computationally not very demanding, would be boring if not designed, i.e., aesthetically structured, to make a totally new type of experience out of what is impossible in the physical world. Imagine a bungy jump over the most enchanting landscape (e.g., the pristine beauty of the Alaskan wilderness). The beauty in question can be computationally very demanding if the representation chosen is iconic. But it can be alternatively suggested in symbolic ways, combining virtual cues, sound, simulated tactile or thermic data. In each of such situations, the modelling, rendering, and animation effort will depend upon what kind of representations are chosen and which dynamic conventions are pursued.

Experience shows that in generating virtual worlds, designers and engineers have to quantify how much realism is necessary in order to make the experience possible. Some applications require no more than cues; others are based on detailed maps; and yet others on various kinds of materials, identified as soft, homogenous or not, buffering or amplifying sound at impact, modifying the Newtonian laws while in free-fall, and so on. This translates into visual requirements of field of view, resolution, of rendering speed, perceptual lag, or in requirements for sound, haptic, and other characteristics. In order to satisfy such requirements within a well chosen type of representation (or synaesthetic combination of different representations), compromises will be made. A higher resolution (for instance, for detail 
relevant to a surgeon) might be chosen over a broader field of view. If compromises are aesthetically balanced, the builders of VR applications will find out that what they need is not a $1000 \mathrm{MHz}$ clock chip and faster buses, but rather a design that combines visual, aural, and tactile representations achieving their optimum through their harmonious integration.

The make believe component of establishing the VR convention is not reducible to the physical characteristics of the display technology, the sound system, the data glove, or of the integrated suit. In an experiment occasioned by the design of an environment for learning and playing dedicated to children (Arketek Ludens, designed by myself and Giuseppe Trogu), we came to the realization that the synergy of various sense data compensates for the extreme requirements of realism (Figure 7).

The procedure applied was inspired by research in experimental psychology and aesthetics [Fechner, et al. (lo)]. It consisted of reducing the amount of visual data while simultaneously introducing sound, haptic, or kinetic elements. If children are immersed in the experience, the reduction of visual 'realism' (e.g., in showing birds, cars, airplanes) in parallel to the increase of associated sound (bird songs, sounds of cars and airplanes) maintains the integrity of the action. The curves resulting from the observations made are rather a qualitative expression of the concern for complementarity of VR representations (Figure 8).

An Interactive Visualizer, like the one built at the Graphics, Visualization, and Usability Center at the Georgia Institute of Technology (11) is not a 'camera' reporting on progress of a work, but a construction metaphor in the first place. As a metaphor, it introduces the user to a world reminiscent of past experiences. This is necessary because to give in to simulation, to become part of it, is cognitively far more challenging than to enter a physical world situation. The metaphor is an aesthetic carrier from one realm to another.

While everything we do in the physical world entails our system of conventions (the symbols of science theories, of designs, of social and political activity), in the virtual world our senses put us in contact with an all encompassing meta-symbol, i.e., dynamic symbol of symbols. Inside this reality, objects are tools that change the world. They can be glyph binders for mapping data variables onto graphic elements, flight simulators, or wands for exploring workrooms where affordance is modelled as a parameter for new designs. At the intersection of the physical world and the limitless

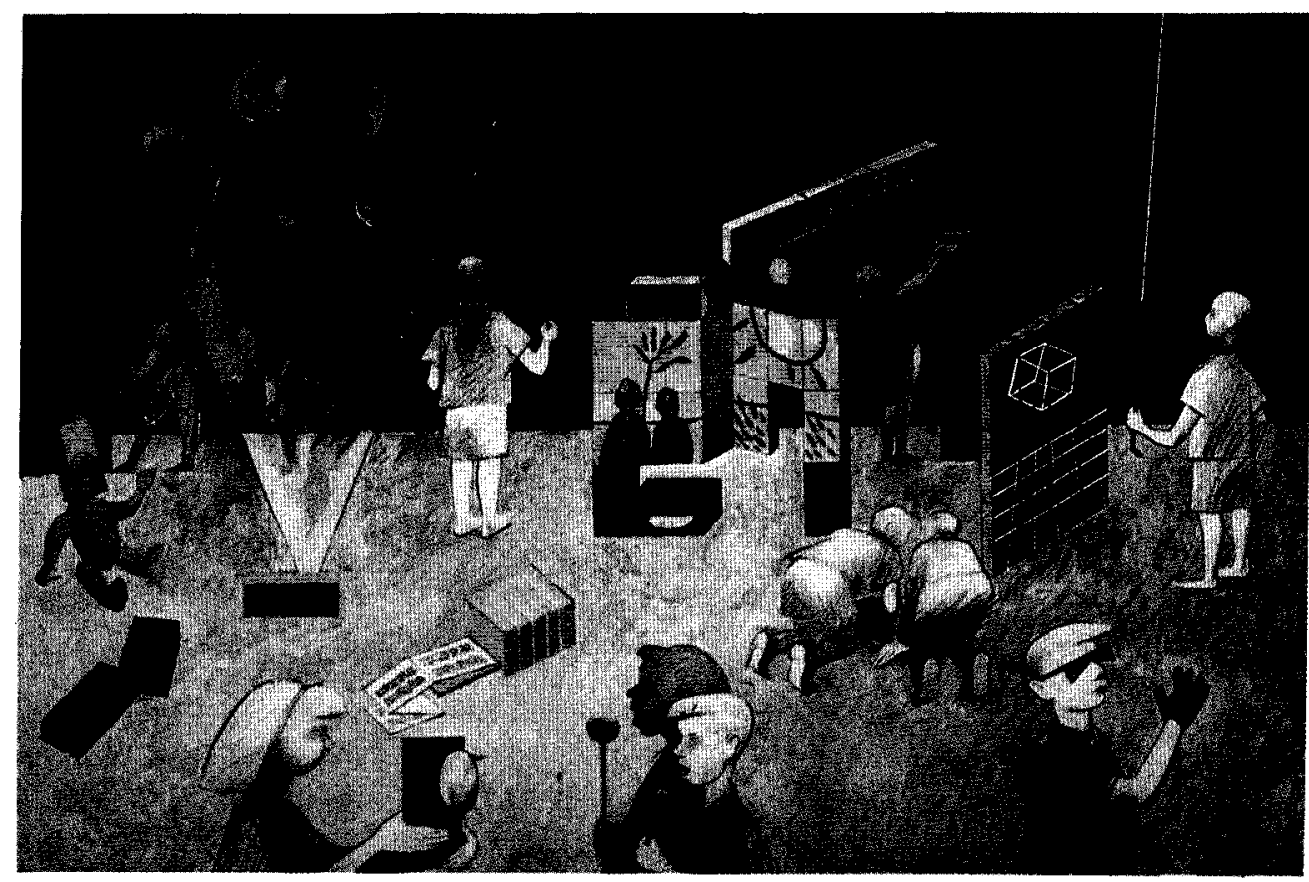

Figure 7. Arketek Ludens (artist rendition). A MINDesign project for the competition Inventing the Future (Japan, 1991). 


\begin{tabular}{c|c|c|c} 
& $\begin{array}{c}\text { Iconic } \\
\text { image }\end{array}$ & $\begin{array}{c}\text { Indexical } \\
\text { image }\end{array}$ & $\begin{array}{c}\text { Symbolic } \\
\text { Image }\end{array}$ \\
\hline $\begin{array}{c}\text { Iconic } \\
\text { sound }\end{array}$ & & & \\
\hline $\begin{array}{c}\text { Indexical } \\
\text { sound }\end{array}$ & 0 & A & \\
\hline $\begin{array}{c}\text { Symbolic } \\
\text { sound }\end{array}$ & 0 & & \\
\hline
\end{tabular}

F igure 8. Representation and make believe. Compensating for visual realism with sound. 0 , make believe established almost without delay; A, make believe established after short learning; $W$, suspension of disbelief requires longer time and is not stable.

imagination of individuals immersed in the virtual reality, a subjective threshold is established. To make things up beyond the threshold is more a matter of imagination than labor. The entrance to the new world is guarded by its aesthetic characteristics, cues to the understanding of what is possible in the computational fiction. The convention of VR is ultimately that of the sensory synergy: how do all the expressive cues make up the new world? In other words, how are the various sensory components involved in constituting the parameters of experience in the new space and time it continuously generates? The perceptual system (which Schlemmer's drawing identifies precisely) and the locomotor system are connected as new sensations, transmitted to effectors, trigger action in the VR environment. Yes, this virtual world is modelled, rendered, animated, musically composed, textured, and danced as behavior is simulated. Accordingly, all of VR is an aesthetic artifact unfolding the many possibilities of the convention or conventions established through the purpose of the application. This conclusion confirms Krueger's early assertions regarding the 'unique melding of aesthetics and technology' in his responsive environments [cf. 3, Introduction, p. xiii].

\section{0 pen-endedness}

Speaking at the National Academy of Sciences (Convocation on Technology and Education, Washington DC, May 10, 1993), Bruce Sterling noted that 'cyber- space is a mirror you can edit.. it's a place (. . .) where you can be promised a rainbow but given a mouthful of ashes'. VR has inspired other well publicized expressive formulations, but few as precise in capturing the difference between aesthetically relevant applications and those living only on the borrowed acceptance of the medium's novelty. There is no doubt that the dominant component of any current VR application is the visual. It does not have to be this way, or at least not for all applications. Knowledge of other sensory channels and the ability to process data pertinent to such channels lead to understanding their aesthetic interinfluence. Their integration is essential for improving the effectiveness of VR applications. It should be noted that the disparity between the immense amount of data that a human being can distinguish (ca. 1040 bits/s) and the relatively limited conscious output (ca. $50 \mathrm{bits} / \mathrm{s}$ ) is in itself significant of the cognitive effort. Aesthetic factors (such as order, symmetry, accent, surprise) allow for the processing of relatively smaller data units with the same results (Figure 9).

The ability and indeed the need to integrate sensory representations other than those characteristic of the visual (sound, haptic, tactile) deserve to be mentioned here again exactly because a proper underlying aesthetics results from the interplay of sensory data. While the core requirement of a VR system is a high performance $3 \mathrm{D}$ image generation capability associated with a complex tracking mechanism and effective simulation programs, processing of sound, tactile, kinetic, and other sensory data can prove as important.

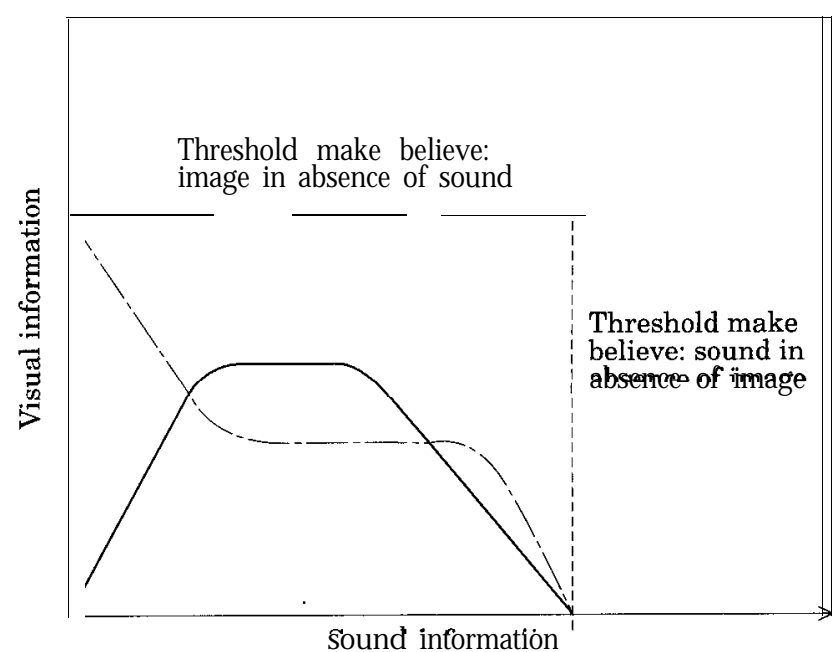

Figure 9. Threshold of make-believe under conditions of image and sound synaesthesia. To date, the diagram captures only qualitative aspects. 
The graphic engine might be pushed to the extremes of its capabilities by, let's say, 'reproducing' a bird in a virtual landscape. Appropriate sound processing will not only ease the job of the visual generator, but introduce the additional information of distance, direction of movement, and even context. As it is already known, the difference in the arrival time and in the intensity of sound to both ears complements 3D visual information. Moreover, shadowing effects (sound travelling around an object) or reflection and absorption effects allow the human being to infer from aural data to spatial configurations. Many times in 'real reality' sensory data complement each other. The aesthetics of VR can only profit from the attempt to transfer this knowledge into the new worlds computationally synthesized. Where field of view, for instance, or the ability to surround the subject, conflicts with requirements of visual acuity, the designer should look for complementary sensory data.

Aesthetic considerations turn into an added constraint (computational overhead) only to the extent that they become a goal in themselves, not the underlying unifying element they are supposed to be. A good example along this line is Rita Addison's Detour: Brain Deconstruction Ahead, a VR system of interaction between imaging science and imaging art presented in the Audio-Visual Experience Environment known as CAVE at SIGGRAPH 94. The environment's immersive and interactive qualities allowed for both a very personal testimony of a photographer's struggle with brain injury after a car accident and for possible therapeutic uses.

The fundamental aspect that such an example makes clear is that through VR applications in which the underlying aesthetic is properly considered, knowledge can be turned into new experiences. Inside the experience many technical limitations might affect the suspension of disbelief, for instance, by influencing the physical condition of the subject. Most trackers used today, for instance, transmit data (position of head, hand, etc.) through a serial line (RS232). At a speed of 9600 baud, a 16-bit word requires a time on the order of $13 \mathrm{~ms}$. Adding all the delays resulting from the transmission of data from trackers, values at which human performance is affected $(50 \mathrm{~ms})$ can be reached. The practical observation is that even with the highest graphics performance, latency can occur and result in simulator sickness. From an aesthetic viewpoint, the trade-off between performance (macro-level) and detail (micro-level) can be handled by implementing synergetic solutions instead of insisting on one aspect -let's say realism-to the detriment of others. Poor coordination of sight, motion, sound, and other sensory data is much more taxing on the overall performance than loss of detail or limited depth complexity. Some technologists still live by a straightforward calculation: a $1280 \times 1024$ pixel screen has $1.3 \times$ lo 6 pixels. In order to ensure a 30 -frame/s display rate, no less than $39 \times$ lo 6 pixels/s need to be produced. At a complexity average of five, the number to consider is almost $200 \times$ lo 6 pixels/s. If two separate displays are used (one for each eye), the number doubles. The system will have to render two separate graphics channels. Adding to all this the rendering information, i.e., amount of detail, shading, transparency, anti-aliasing, i.e., bits per pixel, shows how powerful the hardware has to be. For this reason, real-time (advertised as 'real-life') hardware continues to be designed and made available. Recently, graphics rendering cards processing up to about 400,000 Gouraud shaded Z-buffered triangles per second, achieving a pixel fill rate of almost $1 \times$ lo 9 pixels/s and generating a stereo display were integrated in new commercial products. Dynamic allocations of pixels up to 128 bits per pixel is also an option. Still, the solution is not in such performance, which we of the pioneering age of computer graphics could not even dream of, but in a better understanding of the task at hand.

Appropriate coordination of sensory data and especially a good semiotic strategy of substitution, insertion, and omission change the image quite radically. Complexity can be reduced by substituting image with sound, tactile, haptic, or kinetic information. In many cases, the insertion of indirectly related data, such as direction of movement, allows for lower expectations of detail (fast moving objects blur). Complementing sensory channels usually relieves between 30 and $40 \%$ of the computational effort. No doubt, it introduces the need for alternate imput mechanisms and makes the task of integration more difficult. Even more interesting is the optimization through omission: what and under which circumstances can something be left out without affecting the integrity of the VR experience? There are no standard answers, but there is a lot that can be done in this direction.

Without any exception on record until now, we know that once the VR convention(s) is (are) established, the novelty of the artificial world is not a matter of refresh rates, stereoscopic sophistication, or even scope of application. As a matter of fact, even the most engaging 
VR applications-I can think of the wild ride in the futuristic Cybertron (from Straylight Corp.) or the Fractal Exploratorium - are characterized by a relatively rapid fading away of their novelty. A competitive race among players in the Cybertron proves to be as engaging as the exploration in the Fractal Exploratorium of the evolving universe of galaxies and stars. In less than 10 min (I am being generous!), the novelty was consumed.

As opposed to the uniqueness resulting from the dynamics of change in the physical world, the virtual worlds, as we know them from the examples made public in the last 2 years, usually lack richness and are quite shallow. Granted, VR can be better, and will be many orders of magnitude better in many ways, as we learn more about what it takes to simulate reality. The increased performance of massively parallel machines is only one factor speaking for this optimism. The PDPll used in Psychic Space, or the PC-supported VR attempts (e.g., the distributed model from Sense8 embodied in their Workroom) are difficult to compare to the most advanced research using extremely powerful machines, improved tracking methods, higher quality displays, and better simulation. It is still much too early to speak about maturity, even if many VR applications are deployed in science, presentations, entertainment, and education. Those building them are happy when applications are stable. They all wish for even more CPU cycles, for better I/O and other technical advances in both architecture and programming, especially in real-time operating systems. But what is most important is that together, we all learn that we need to know more about the human being, especially about its cognitive condition, in order to facilitate VR experiences. We know quite well, for instance, that the interplay of combined sensorial perceptions is of critical importance. But does it suffice to provide an optimum on each communication channel? How relevant are the characteristics of visual monocular or stereoscopic communication (bandwidth, dynamic range, transmission or delay, signal-noise ratio, resolution)? Or those of the haptic or audio channels? Isn't it true that the interplay of data is almost insignificant?

The 90-95\% resolution of vocal/synthetic speech, i.e., recognition in a 50,000-word vocabulary is probably correct if we deal only with the synthetic sound. But if, by design, we combine synthetic speech and tactile information, or images, the number will decrease. Exactly in this area, of providing a precise but expressive interplay are aesthetic considerations determinant. The power of association, of suggestion, the power of syncretism- all of aesthetic condition -makes the difference. Various experts will always argue from an insular perspective. Aesthetic integration transcends specialized insularity. William J. Grosky (12) put the issue of maturity in a scientific field in a very convincing perspective: 'One sign that a field $\mathbf{F}$ has matured is when most of its research problems consist of such a complex mix of information from its component areas F 1,.. Fn, that their solution requires the integrated expertise of researchers trained in field $\mathrm{F}$, not merely a research group of specialized researchers in fields $\mathbf{F} 1, \ldots \mathbf{F n}$, some of whom may not believe in the viability of the field to begin with'. This integrated expertise is far from emerging in a coherent theory, and even less in coherent products (Figure 10).

The underlying aesthetics for supporting open-ended interactions within a VR application is not reducible to good graphics, high performance modelling or rendering, simulation algorithms for textures, or signal processing for realistic sound renditions. We have many experts in component areas who can provide good (or always good) real-time animation or tracking. What we do not have is the deeper understanding of what it takes to generate worlds based on the integration of various sensory data. The experience of hearing completes that of seeing, and under certain circumstances can even substitute for it. VR applications that integrate this and similar knowledge, in fact integrate aesthetic components of a constitutive, not descriptive, nature. Progress in three-dimensional virtual acoustic environments is no less relevant to supporting openended interactions than the attempts to provide haptic feedback (addressing the issue of the tactile), or to integrate smell as a component of VR. The aesthetics of the world is, after all, a unifying framework. As long as we keep dealing with partial aspects of virtual worlds, we will report technological progress, but no real cognitive breakthrough. While speech synthesis is still quite bad, while force feedback is still rudimentary, while slow or unoptimized update creates lag problems, and so on, we can point to particular, yet unresolved, technological problems. But let's imagine that we have everything already figured out. Would perfect speech synthesis, and force feedback, and appropriate phase and speed update give VR the support for open-ended interactions? Probably not, exactly because the domain is not the sum total of its components, but rather a qualitative synthesis 'glued' together by its aesthetic condition. 


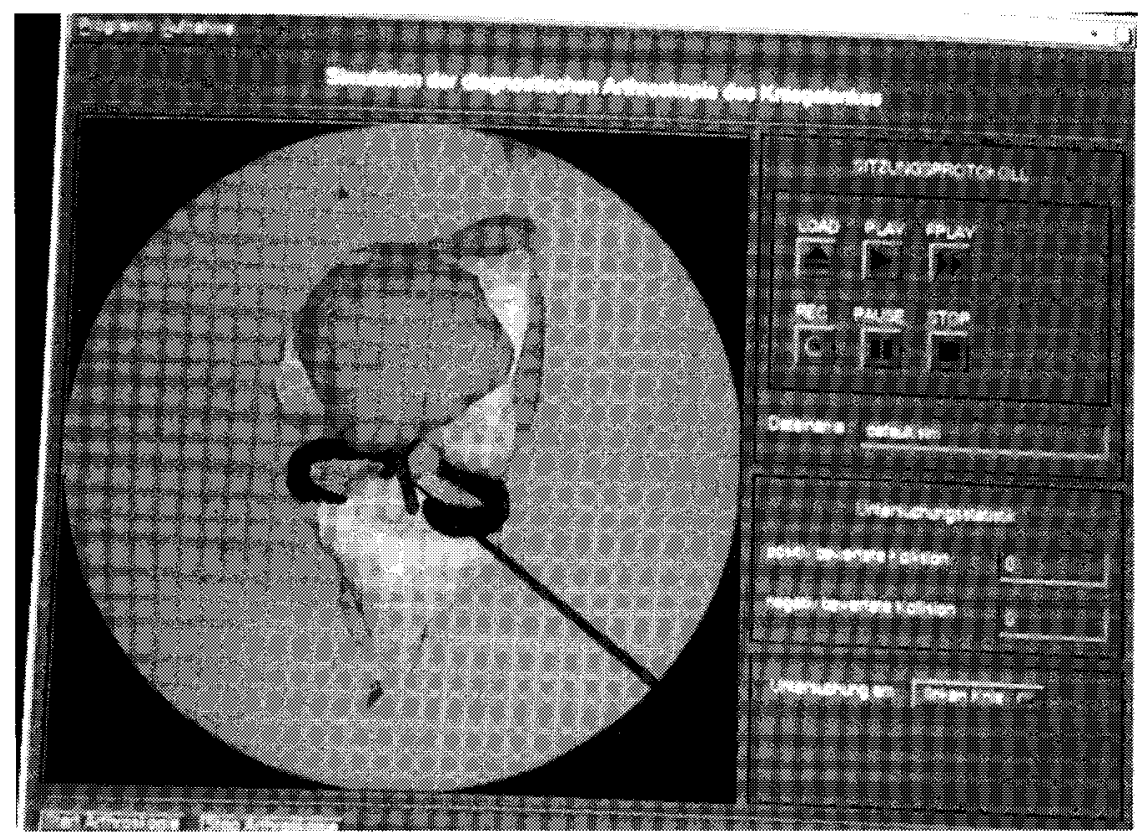

Figure 10. Training simulator for arthoscopy. A user interface image from the VR Center of the Frauenhofer Institut für Graphische Datenverarbeitung (Darmstadt, Germany).

VR is syncretic in nature. It has to scale as the experience of the respective application scales. Knowing that cognitive processes are non-linear in nature, this scaling, too, will have to be non-linear. And if this is not enough, it probably needs an evolution component, a self-learning mechanism. If we can reach such a level, the novelty of VR will be less subject to fading away. Make believe is not a matter of appearance; it is one of functioning. To see the world through someone else's eyes, to become a lobster or to simply step down steep stairs in a VR world are impressive examples of computational progress. So were the flight simulators of the NASA-AMES pre-VR age; and before, the teieoperation technology, vehicle simulation; and so are many VR entertainment products.

Let us put the issue in the cultural context to which it belongs. The replica to the Grotte de Lascaux (the Lascaux cave) is an example of high-degree texture simulation, realistic depth perception, real-time tracking allowing for unlimited speed of user motion, and many (up to 100) interactive viewers. The only shortcoming is that it does not involve computation. And that it is limited to an unextendable database, that of the original prehistoric cave! So the question to pose is: What justifies our effort to create techniques that apply computation to the generation of valid cognitive realities? The ideal answer is too good to be true: such realities translate knowledge into new experiences. The replica to the Lascaux cave does not have this quality. VR applications engage us in ways no other 'reality' can. And through this engagement, we can gain further access to knowledge, we can inquire about ourselves, we can interact. we can be entertained.

Nevertheless, the reality looks a little different. Because of their closed nature, VR applications are not yet a medium that effectively engages those immersed in them. Individual experiences do not scale up to collective experiences. The missing emotional component could be effectively supported if the VR experiences were anchored not in the sensorial alone, but in the aesthetically cultivated senses, i.e., in culture. No surprise, then, that at the level of drives (sexual, foremost), VR is just enough to make possible imaginative experiences. But also no surprise that in the absence of aesthetically integrated elements that could affect feelings, the 'engagement' is rather tedious, if it is established at all. A critical component would give them motivations in extension of scientific and technological progress.

\section{VR Negotiated}

Immersion sounds better than what VR actually offers. 
The more accurate term for the VR experience is 'negotiation'. One starts in the physical world, puts on a helmet and gloves, goes through some adaptations. Cognitively, this creates a forceful barrier to the desired make-believe. Disbelief is built up as an individual prepares for VR. The suspension of disbelief (which not only the authors of CAVE made their objective) results from the process of establishing the convention of the meta-symbol, i.e., expressed in the representation chosen and in the context of interpretation. After the convention is in place, it needs to be maintained as a coherent aesthetic framework. Interactions test the convention, and the more these are tested, the more intense the VR experience becomes. An alternative to the metaphor of immersion is the more factual notion of negotiation. Indeed, in VR we negotiate in a space and time different in nature from those of the physical world. Negotiations in VR do not imply explicitness. They carry conceptual entailments. Among these, the right to refuse, to ignore, moreover 'to end the game' are essential. At first this seems easy ('Pull the plug!'), but the more you think of it, the more you understand that make-believe is successful only if the suspension of disbelief is hard to attain; that is, if we are carried away as drug addicts are in their use of one substance or another.

Some aesthetic experiences are such that the borderline between the physical world and the world of art and artifice is clearly defined: frames for pictures, the falling curtain in a theatrical performance, the credit list prior to switching the lights on after a movie. The viewer, no matter how immersed in the drama, remains outside, can leave the theater as one would leave a museum or a concert. As negotiator of VR, the individual is made part of it. This is why there is no meaningful looking over one's shoulder in VR. You are in or you are out, like in a maze! (Or again, like in the drug experience, by no accident associated to VR.) Data are embedded in the virtual world, object representation and data structure can be identical, eyehand (and generally eye-limbs, body parts) coordination needs to be carefully assured. Any incongruence can cause perceptual confusion. Ending a VR negotiation is no less taxing than walking on the moon's surface, or returning from a drug 'trip'. In fact, since a world of characteristics different from those of the physical world (such as the gravity-free moon) is simulated in VR, and since to negotiate this world engages sensory and mental resources, there is a need to design this world in ways that allow for escape, indeed for cognitive extradition. It is not enough to monitor, even with a 6D position tracker, the coordinates of one's head or arms and realign the virtual world to keep its appearance. The result might be a behavioral map, but the background processes are more important. Accordingly, cognitive states need to be monitored so that, for instance, destructive effects do not ensue. Interestingly enough, cues to something that can go wrong or went wrong are connected to the underlying aesthetics. Blurred vision (due to nausea), lack of balance, orientation difficulties correspond to modifications in the syncretic world of images, movements, sounds, textures, etc. In this artificial world, more is not the answer, but usually the problem. The gap between the virtual and the real is similar to that between complicated and complex.

In effect, the end of the game is the end of the convention. Aesthetic strategies to this effect consist of slow reintegration of the individual in the physical world by means of juxtaposition. As unimportant as this may sound to some, it is not only a matter of respect for the integrity of the human being, but also a matter of specific knowledge. The subjective threshold is passed twice in the VR experience-once on entry and establishing the convention; and again on exit. And as we know by now, this threshold has quite a hysteresis due to the fact that there is quite a difference between sensorial levels and the levels of effecting.

The world of VR knows no decay and is quite dense. Those inside are separated from those outside. We experience ourselves in VR, and in this experience we are guided by the logic of our senses. This is why the underlying aesthetics plays such a fundamental role. The individual negotiating VR completes all that which, with relative crudeness, defines another world. Synaesthesia makes illusion possible. This being the case, the aesthetics in question is that of illusion. Different as they are, the illusion experienced by a quadriplegic with sensors attached to active body parts (tongue, for instance) to compensate for the atrophy of other parts, and the illusion experienced by a molecular chemist, an astronomer, a surgeon, or an architect are, after all, illusions. The objectivity of the physical world is recreated, moreover experienced in a subjective environment. This is why the processing of data in VR is effective as a means of embodying, in image, sound, haptic, kinetic, highly correlated multivariate data from large-scale computation. Visual characteristics - position, shape, size, orientation, color - as well as characteristics of sound, motion, force, and texture can be associated to data. The mapping of variables to the VR 
synthesis is to a certain degree analogous to an exercise in creative visualization. Real-time imagery and interactivity enforce the feeling of authenticity. Each change in the convention allows a different interpretation. The fact that this is more a quality than quantity calculus should not surprise. Some 25 years ago, Zemanek (13) came to understand computer science as 'the engineering of abstract entities'. VR confirms this definition in many ways. What we learn from here, and from similar VR applications that do justice to the underlying aesthetics, is that to negotiate VR means to edit the world to which the VR subject is sensorially connected in real time. The medieval model that all knowledge comes from the senses is turned upside down in the virtual world, where through the experience new knowledge is 'injected' into the sensorial. VR feeds senses with the products of human imagination and illusions, and what results is the intense living of the meta-symbolic artifact. In the meta-symbolic, the newly engineered abstractions making up that world are cognitively authenticated.

\section{Acknowledgments}

The author would like to thank. in addition to the anonymous reviewers, Dr. Martin Göbel of the Frauenhofer Institut für Graphische Datenverarbeitung (Darmstadt, Germany), Stephen Ellis, Ph.D., Head, Advanced Displays and Spatial Perception Group, NASA, Ames Research Center. CA; Professor Joachim Sauter of Art + Corn. Berlin; Baruch Gorkin of Access Factory, NY: Pino Trogu.
San Francisco; Anabel1 Loewe, Ute Hilgers, Peter Peltsarszky. and Peter Stefan of the Computational Design Institute, and Hans Keller of the Electrical Engineering Department, University of Wuppertal; and Piroshka Queen for their making available images, diagrams, information, and exchange of ideas for this article. The copyright for all images rests with the persons and organizations that made them available.

\section{R eferences}

1. Cruz-Neira, C. Sandin, D.J. DeFanti, A.T. Kenyon. R.V. \& Hart, J.C. (1992) The Cave. Audio visual experience automatic virtual environment. Comm. ACM. 35(6): 65-72.

2. Jenny Holzer (1993) Guggenheim Museum. 1993.

3. Krueger, M.W. (1984) Artificial Reality, Reading. MA: AddisonWesley.

4. Ellis, S.R. (1994) What Are Virtual Environments? IEEE Comput. Graphics Appl., 14(l): 17-22.

5. Baumgarten. A.G. (1970) Aesthetica (reprint of original 1750 edition, Frankfurt a. d. Oder), Hildeshim: Georg Olms Verlag.

6. Nadin, M. (1986) Visual semiosis applied to computer graphics. In Annual Conference Proceedings of the ASEE, Hanover PA: The Sheridan Press.

7. Heim, M. (1993) The Metaphysics of Virtual Reality, New York: Oxford University Press, p.29.

8. Gibson. J.J. (1986) The Ecological Approach to Visual Perception, Hillsdale. NJ: Lawrence Erlbaum Associates.

9. Nadin, M. (1986) Simulation Machine. Eminent Scholar in Art and Technology Research Program, Ohio State IJniversity.

10. Fechner. G.T. (1978) cf. Vorschule der Aesthetik, 192.5. A reprint of the original edition was provided by Olms Verlag of Hildesheim and New York. $674 \mathrm{pp}$

11. Ribarski, W. Bolter. J. op den Bosch, A. \& van Teylingen. R. (1994) Visualization and analysis using virtual reality, IEEE Comput. Graphics Appl., 14(l): 10-12.

12. Grosky, W.J. (1994) Multimedia information systems. IEEE Multimedia, 1( 1): 22 .

13. Zemanek, H. (1971) Was ist Informatik? Elektronische Rechenanlagen, 13(4): 154-171. 